\title{
Dirección Estratégica en las Superintendencias del Estado de Chile
}

\author{
Ana M. Barra \\ Facultad de Ciencias Empresariales. Universidad del Bío-Bío. Avenida. Collao 1202. Casilla 5-C. Concepción. Chile. \\ (e-mail: abarra@ubiobio.cl)
}

Recibido Sep. 15, 2016; Aceptado Nov. 23, 2016; Versión final Dic. 21, 2016, Publicado Jun. 2017

\begin{abstract}
Resumen
El objetivo de este trabajo es realizar un análisis comparativo de las Superintendencias del Estado de Chile, respecto al proceso de Dirección Estratégica y sus componentes, con el fin de diagnosticar problemas y proponer algunas mejoras. En las organizaciones del sector público donde el Estado hace cumplir la política expresada en las leyes que hay en el país, a través del poder legislativo, ejecutivo y judicial, se observa que existen algunas diferencias en cuanto al grado de avance del proceso de Dirección Estratégica que estas instituciones han formulado para desempeñar sus funciones. Esta situación ha motivado el estudio que se presenta. Se utiliza como metodología la revisión bibliográfica de las variables, etapas y componentes del proceso de dirección. Se revisan las estructuras de cada superintendencia, se adoptan algunos criterios para analizar falencias y realidades. Los resultados permiten elaborar algunos criterios de comparación de las Superintendencias, dando como respuesta un diagnóstico y proponiendo mejoras al proceso de Dirección Estratégica
\end{abstract}

\section{Strategic Direction in the Superintendent Offices of the State of Chile}

\begin{abstract}
The objective of this work is to do a comparative analysis of the Superintendent Offices of the State of Chile, about the process of Strategic Management and its components, to diagnose problems and propose improvements. In public organizations where the State complies and enforces the policy or will expressed in the laws of the country through legislative, judicial and executive power, it is observed that there are some differences in the level of progress in the process of Strategic Management that these institutions have formulated or implemented to perform their functions. This situation has motivated the study presented here. The methodology consists of a bibliographic review of variables, stages, and components of the management process. Every Superintendents Office and some criteria from relevant authors are adopted in order to completely understand every variable of the process and analyze its weaknesses and realities. The results allow elaborating some criteria for comparing the Superintendents Offices of the State of Chile, giving as a response a diagnostic, proposing improvements to the process of Strategic Management.
\end{abstract}




\section{INTRODUCCIÓN}

Las empresas hoy en día se mueven en un contexto extremadamente difícil, en el que son muchos y muy valiosos los competidores, en el que ya no basta crear un empresa para tener éxito, ni siquiera basta con saber vender. Por otra parte, el entorno es cambiante, turbulento, las fronteras económicas entre los países han ido desapareciendo, existe competitividad, globalización de la economía, ventajas competitivas, mejora de la calidad, reducción de costes y cultura empresarial (Navas y Guerras, 2007). Por tanto, se hace cada vez más necesario para los empresarios y altos directivos de las empresas dedicar un mayor esfuerzo y análisis a la elaboración y elección de la estrategia más adecuada para responder a los retos del entorno y hacer así a la empresa más competitiva en este difícil contexto.

En algunos países, el gobierno ha impulsado el proceso de modernización de la Gestión Pública, que, involucra la renovación y recuperación de las instituciones públicas con el fin de prestar servicios a la ciudadanía bajo estándares establecidos de calidad, en forma oportuna y con especial cuidado en lo que dice relación al trato hacia los usuarios. Este proceso de modernización del estado, estuvo marcado por una visión gerencialista, con énfasis en la eficiencia, en un estilo de gestión orientado a resultados y en la aplicación de modernos principios de gestión, procurando el funcionamiento regular y eficiente de los servicios, junto con respetar los principios de transparencia, equidad y disciplina fiscal. Considera además, y de manera prioritaria la satisfacción de los usuarios, la realización personal y profesional de los funcionarios, la asignación eficiente de los recursos y la capacidad de respuesta ante las nuevas demandas de la sociedad.

Para que lo anterior se cumpla, se pone énfasis en la transformación de la cultura organizacional que caracteriza a la administración pública. Lo fundamental es dar un giro en los hábitos, costumbres y forma de hacer las cosas en los servicios públicos, incorporando herramientas innovadoras y prestaciones con altos estándares de calidad y con una mejor atención hacia los usuarios. En la actualidad el desafío de las empresas es cada vez mayor, tanto para el sector privado como el público. El surgimiento de un nuevo escenario a nivel mundial y local es cada vez más competitivo, esto ha significado un replanteamiento en el proceso de Dirección Estratégica de las instituciones. Además, el desarrollo de nuevas tecnologías de información ha provocado un gran impacto en la capacidad de creatividad empresarial, tanto en la fase de generación productiva como en la de los servicios. Bajo este escenario la Dirección Estratégica contribuye a que las organizaciones logren alcanzar sus objetivos y tengan un mejor resultado.

Por ello, la Dirección Estratégica no sólo es importante en las instituciones privadas, sino también, en las instituciones públicas, las que han empleado distintas herramientas o programas para optimizar sus recursos y procesos. El objetivo de este trabajo es realizar un análisis comparativo de las Superintendencias de Chile con el fin de diagnosticar, analizar y proponer mejoras al proceso de Dirección Estratégica, además de conocer el proceso de Dirección Estratégica de las Superintendencias del Estado de Chile y analizar los Componentes del proceso de Dirección Estratégica de cada una de las Superintendencias del sector público, con el fin de visualizar el estado de avance del proceso en estas grandes organizaciones.

Es un proceso cuyo objetivo principal es definir una estrategia y ponerla en práctica, siendo tan importante la formulación como la implantación de la misma. Es dinámico y continuo pues el entorno exige estar a la expectativa de cualquier cambio Álvarez (2007).

Según los autores Johnson et al. (2006), describen el proceso de Dirección Estratégica en tres etapas: el análisis estratégico, la elección estratégica y la implantación de la estrategia. Rumelt et al. (1994), consideran que la Dirección Estratégica incluye aquellas materias que son de principal interés para la dirección general o para quien pretenda investigar las razones del éxito o fracaso de las organizaciones. El autor Eduardo Bueno (1996), se refiere a este proceso como "el sistema de dirección que pretende identificar de forma precoz los cambios externos e internos para instrumentar respuestas rápidas, procurando que el nivel de sorpresa de los impactos sea el menor posible".

La Dirección Estratégica es un estilo de dirección que pone el énfasis en la identificación precoz de los cambios externos, como respuesta al dinamismo y complejidad crecientes del entorno empresarial. Los autores Navas y Guerras (2007), plantean un proceso de dirección estratégica compuesto de tres partes fundamentales: el análisis estratégico, las opciones estratégicas y la implementación y control de las estrategias. Johnson, Scholes y Whittington (2006), definen algo similar a los autores Navas y Guerras (2007), refiriéndose los primeros al análisis estratégico como el conocimiento de la posición estratégica de la organización, la elección estratégica en la formulación de una serie de estrategias a seguir, evaluarlas y elegir alguna de ellas y la implantación de la estrategia como la puesta en práctica la estrategia elegida en la empresa y conducir los cambios que se presenten en la organización. 
Se adoptará el modelo de Navas y Guerras (2007), donde se explicarán las tres etapas del proceso para posteriormente aplicarlas a las superintendencias en estudio. La etapa del Análisis Estratégico se compone esencialmente del estudio de tres elementos que conjuntamente determinan el espacio estratégico en el que se va a desenvolver la empresa y que serán los ejes sobre los cuales se construirá su estrategia: la definición de los fines y misiones generales de la empresa, el análisis externo y el análisis interno. La visión identifica las diferencias entre la situación actual y la deseada y, por consiguiente, marca la dirección a seguir de la empresa. En este mismo sentido, para F. David (2003), la declaración de visión debe responder a la pregunta ¿qué queremos llegar ser?

El autor Paredes (2006), propone una serie de elementos a tener en cuenta en su redacción: Dimensión de tiempo, Ser amplia y detallada, Positiva y alentadora, Realista en lo posible, Proyectar sueños y esperanzas, Incorporar valores e intereses comunes, Debe ser difundida interna y externamente. La misión recoge la razón que justifica la existencia de la empresa, constituyendo una declaración de principios mediante la cual la empresa se presenta ante la sociedad. Es importante por tanto que la misión sea conocida por todos los miembros de la organización, ya que sirve como elemento de identificación con la filosofía de la empresa y de cohesión entre todos los empleados, Álvarez (2007). Según el autor Hax y Majluf (1997), la misión se debe hacer a partir de las siguientes variables: la definición del campo de actividad de la empresa, la identificación de las capacidades esenciales, debe ser posible, amplia y concreta.

Los objetivos se tienen que fijar según determinados criterios y, además, cumpliendo unas condiciones si se quieren alcanzar con éxito: (i) Deseables: porque deben ser expuestos por alguien como propósito o deseo; en caso contrario, será una simple rutina; (ii) Factibles: porque tiene que ser posible alcanzarlos; (iii) Cuantificables: necesarios para control, adecuada asignación de responsabilidades; (iv) Comprensibles: porque tendrán que ser ejercidos, evaluados y controlados; (v) Motivadores: porque una definición y valoración adecuada puede dar lugar a una satisfacción al llegar a ser realizados.

\section{Análisis Externo}

El entorno general se refiere al medio externo que rodea a la empresa desde una perspectiva genérica, es decir, a todo lo que rodea a la empresa derivado del sistema socioeconómico en el que desarrolla su actividad. La variable político-legal corresponde a una de las variables que tienen mayor impacto sobre las Superintendencias en estudio, principalmente por ser instituciones públicas, autónomas, fiscalizadoras y reguladoras, que se relacionan con el Gobierno a través de sus Ministerios y su jefe superior, el Superintendente, quien es nombrado por el Presidente de la República.

\section{Análisis Interno}

Este análisis es ciertamente de carácter muy general y su objetivo es determinar el tipo y características fundamentales de la empresa. Conocer la identidad de la empresa ayuda a entender mejor el soporte estratégico fundamental para su actuación competitiva, Navas y Guerras (2007). En la segunda etapa de la Formulación de las Estrategias, la empresa busca opciones válidas para conseguir los objetivos. Así, la empresa debe plantearse cómo competir mejor, cómo desarrollar sus actividades en el futuro, cómo mejorar sus capacidades internas o como responder a las actuaciones de otros competidores o agentes económicos o sociales, Navas y Guerras (2007). Hitt et al. (1999) y Mintzberg, y Lampel (1999), coinciden en que la formulación de estrategias es la parte en donde se recopila la información de las empresas identificando las oportunidades y amenazas del entorno y las fortalezas y debilidades de la compañía. Con este conocimiento, las empresas elaboran sus propósitos estratégicos, aprovechando todos sus recursos, capacidades y aptitudes para consolidarse como una compañía competitiva.

Referido a la tercera etapa de Implantación de Estrategias y Control, el autor David [6] se refiere a la etapa donde se produce el cambio y el verdadero trabajo del proceso estratégico. Los autores Johnson y Scholes (2006), afirman que la implantación de la estrategia se ocupa de cómo traducir la estrategia en acción organizacional mediante el diseño y la estructura de la organización, la planificación de recursos y la gestión del cambio estratégico. El proceso de control estratégico debe observar también el ajuste organizativo, es decir, la compatibilidad entre la estrategia y el soporte organizativo; la estructura, sistema de dirección o cultura de la empresa, Schendel y Hofer (1979)

\section{METODOLOGÍA}

La metodología utilizada para el análisis comparativo respecto de la Dirección Estratégica de las Superintendencias del Estado de Chile, es mixta, por cuanto incluye características de tipo exploratoria y descriptiva. Exploratoria, por cuanto el análisis a realizar ha sido poco estudiado y las ideas del tema son vagas, por tanto permite familiarizarnos con fenómenos relativamente desconocidos. Descriptiva debido a 
que el propósito del estudio es describir situaciones, escenarios, realidades, para ver cómo se manifiesta el proceso de Dirección Estratégica en las distintas organizaciones sujetas de análisis. La metodología obedece a la aplicación del modelo de Dirección Estratégica de Navas y Guerras (2007), a través de un cuestionario aplicado a 8 de 9 superintendencias que constituyen la muestra, y tiene trece preguntas de las cuales una es abierta, tres de alternativas y nueve, una combinación de ambas, que permite fundamentar y especificar la respuesta. Las preguntas abiertas y mixtas se realizaron con el objetivo de profundizar las respuestas cerradas obtenidas, y dicen relación con el estado en que la organización realiza Dirección Estratégica y quiénes participan en dicho proceso, cuáles son las herramientas que utilizan para recabar la información con la finalidad de elaborar el plan y quiénes la implementan o la ponen en marcha.

En cuanto a la información necesaria para el trabajo, proviene de fuentes primarias y secundarias. La información primaria se obtuvo a través de un cuestionario aplicado a las Superintendencias objeto del presente estudio con la finalidad de obtener los datos relacionados con el proceso de Dirección Estratégica. El instrumento consta de trece preguntas de las cuales una es abierta, tres de alternativas y nueve, una combinación de ambas, que permiten fundamentar y especificar la respuesta. El cuestionario se aplicó a los Encargados de las Unidades de Atención Ciudadana, Jefes de Unidades de Control de Gestión, Jefes de Desarrollo Corporativo, Jefes de Área de Gestión de Proyectos Estratégicos, Jefes de División de Gestión y Recursos y/o Jefes de Comunicaciones de cada una de las Superintendencias. El número cero corresponde a respuesta afirmativa y el uno a respuesta negativa. La información secundaria se obtuvo a través de la recopilación de información obtenida desde las páginas web de las organizaciones sujetas a estudio, además de sus respectivas cuentas públicas, entre otros.

\section{RESULTADOS}

El universo objeto del presente estudio, corresponde a las nueve Superintendencias de Chile, que se encargan del control y de la vigilancia de determinados sectores económicos o sociales del país y que son: Superintendencia de Bancos e Instituciones Financieras (SBIF), Superintendencia de Casinos de Juego (SCJ), Superintendencia de Electricidad y Combustibles (SEC), Superintendencia de Pensiones (SIP), Superintendencia de Quiebras, Superintendencia de Salud (SIS), Superintendencia de Seguridad Social (SUSESO), Superintendencia de Servicios Sanitarios (SISS), Superintendencia de Valores y Seguros (SVS). El tamaño de la muestra para el universo, es ocho, por cuanto se excluyó la Superintendencia de Quiebras, sobre la base de que era la organización que contaba con menos información tanto en su sitio web, cuenta pública y otros, proporcionando antecedentes mínimos para llevar a cabo el estudio.

\section{Análisis de la Visión}

No todas las Superintendencias en estudio poseen visión, las que corresponden a la Superintendencia de Casinos y Juegos y la Superintendencia de Electricidad y Combustible. Para analizar esta directriz estratégica, se ha establecido la siguiente categorización, en base a los componentes definidos por Paredes (1996), ver Figura 1. En la tabla 1 se detalla el resumen de los componentes de la Visión por Superintendencias, no se muestra SCJ ni SEC, pues no presentaron respuesta.

\section{Análisis de componentes de la visión de las Superintendencias de Chile}

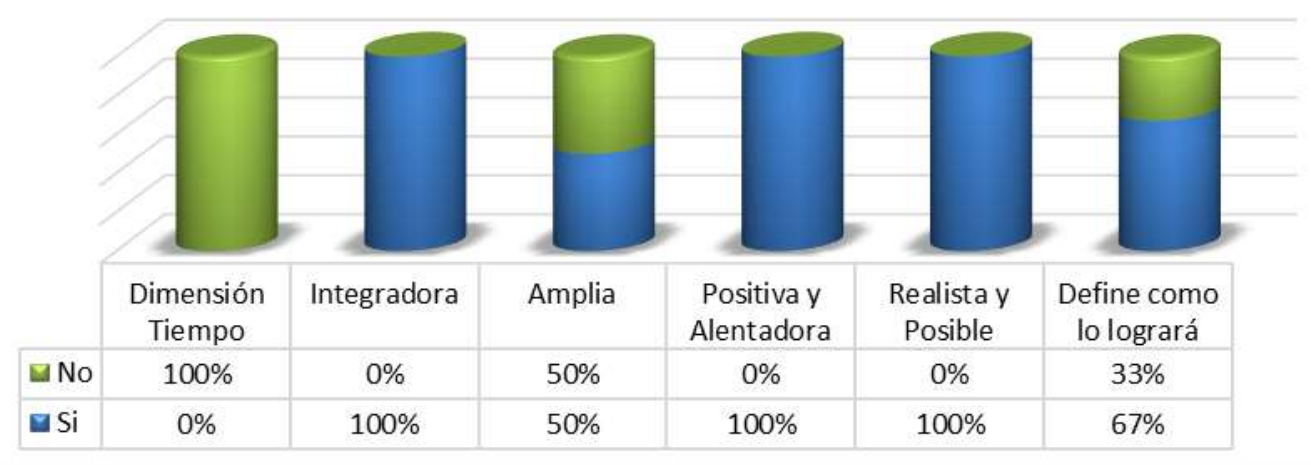

Fig. 1: Análisis de componentes de la visión de las Superintendencias de Chile. 
Tabla 1: Componentes de las Superintencias Chilenas.

\begin{tabular}{|l|c|c|c|c|c|c|}
\hline \multicolumn{1}{|c|}{ Componentes } & SBIF & SIP & SIS & SUSESO & SISS & SVS \\
\hline Dimensión Tiempo & 1 & 1 & 1 & 1 & 1 & 1 \\
\hline Integradora & 0 & 0 & 0 & 0 & 0 & 0 \\
\hline Amplia & 1 & 1 & 0 & 0 & 1 & 0 \\
\hline Positiva y Alentadora & 0 & 0 & 0 & 0 & 0 & 0 \\
\hline Realista y Posible & 0 & 0 & 0 & 0 & 0 & 0 \\
\hline Define como lo logrará & 0 & 1 & 0 & 0 & 1 & 0 \\
\hline
\end{tabular}

\section{Análisis de la Misión}

Cabe señalar, que la totalidad de Superintendencias objeto de estudio, poseen Misión y para realizar su análisis se ha establecido la siguiente categorización, en base a los componentes definidos por Hax y Majluf (1997), ver Figura 2. En la tabla 2 que se detalla a continuación, se individualizan los componentes de la Misión, por cada una de las organizaciones en estudio.

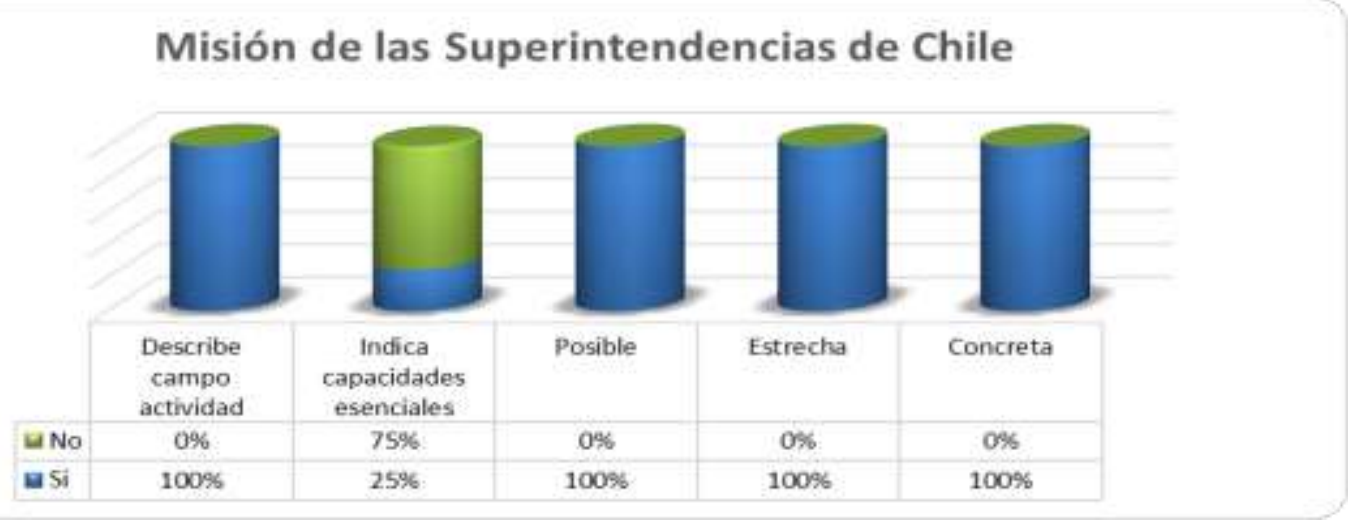

Fig. 2: Misión de las Superintendencias de Chile.

Tabla 2: Individualización de los componentes de la Misión.

\begin{tabular}{|l|c|c|c|c|c|c|c|c|}
\hline \multicolumn{1}{|c|}{ Componentes } & SBIF & SCJ & SEC & SIP & SIS & SUSESO & SISS & SVS \\
\hline Describe campo de actividad & 0 & 0 & 0 & 0 & 0 & 0 & 0 & 0 \\
\hline Indica capacidades esenciales & 0 & 1 & 1 & 1 & 1 & 0 & 1 & 0 \\
\hline Posible & 1 & 0 & 0 & 0 & 0 & 0 & 0 & 0 \\
\hline Estrecha & 0 & 0 & 0 & 0 & 0 & 0 & 0 & 0 \\
\hline Concreta & 0 & 0 & 0 & 0 & 0 & 0 & 0 & 0 \\
\hline
\end{tabular}

\section{Análisis de los Objetivos}

La totalidad de las organizaciones estudiadas, poseen objetivos estratégicos y para realizar su análisis se ha establecido la siguiente categorización, en base a los componentes definidos por Hamel y Prahalad (1990). Ver Figura 3.

\section{Análisis de los objetivos Estratégicos}

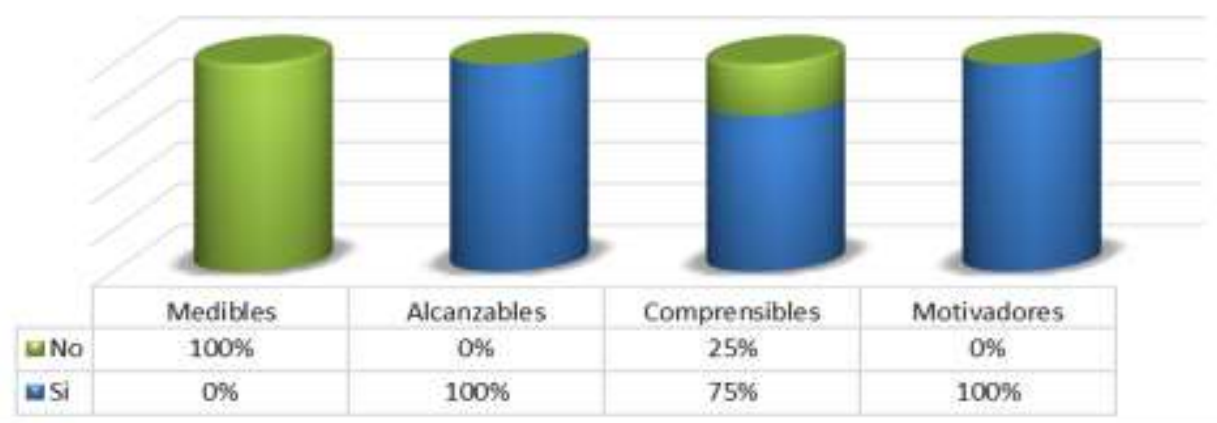

Fig. 3: Análisis de los objetivos Estratégicos. 
Medibles: Cabe destacar que las Superintendencias no cuentan con objetivos medibles. Alcanzables y motivadores: Se destaca que la totalidad de las instituciones examinadas, poseen objetivos alcanzables y motivadores. Comprensibles: Sólo el $75 \%$ de las organizaciones posee objetivos comprensibles. En la tabla 3 , se detallan los objetivos de cada una de las Superintendencias, con sus componentes. En relación a las personas que participan en la formulación de las Directrices estratégicas, el $75 \%$ de los entrevistados coinciden que quienes las desarrollan, son los directivos. Solo la Superintendencia de Salud afirma realizarlo de manera conjunta con el aporte de toda la organización.

Tabla 3: Objetivos de las Superintendencias de Chile.

\begin{tabular}{|l|c|c|c|c|c|c|c|c|}
\hline \multicolumn{1}{|c|}{ Componentes } & SBIF & SCI & SEC & SIP & SIS & SUSESO & SISS & SVS \\
\hline Medible & 1 & 1 & 1 & 1 & 1 & 1 & 1 & 1 \\
\hline Alcanzables & 0 & 0 & 0 & 0 & 0 & 0 & 0 & 0 \\
\hline Comprensible & 0 & 0 & 0 & 1 & 0 & 0 & 1 & 0 \\
\hline Motivadores & 0 & 0 & 0 & 0 & 0 & 0 & 0 & 0 \\
\hline
\end{tabular}

\section{ANÁLISIS ENTORNO GENERAL}

\section{Factores económicos}

Las Superintendencias chilenas, por tratarse de empresas públicas, no se ven muy afectadas por los indicadores económicos, evolución de la renta o niveles de inflación. No obstante, se puede mencionar que eventualmente en caso de recesión económica, podría influir negativamente en su presupuesto anual.

\section{Factores político legales}

Chile es un país políticamente estable el cual se ha posicionado como el país mejor rankeado a nivel latinoamericano, información proveniente de organizaciones internacionales que evalúan a los países según su nivel de competitividad global, libertad económica, transparencia y corrupción, y es el segundo país mejor evaluado que presenta mejores condiciones, en relación a otros, para realizar negocios. En este contexto, los factores político-legales, corresponden a una de las variables que tienen mayor impacto sobre las Superintendencias en estudio, principalmente por ser instituciones públicas, autónomas, fiscalizadoras y reguladoras, que se relacionan con el Gobierno a través de sus Ministerios y su jefe superior, el Superintendente, quien es nombrado por el Presidente de la República. En este mismo sentido, las Superintendencias se ven sometidas a la fiscalización de la Contraloría General de la República.

\section{Factores socioculturales}

Las variables que influyen en las Superintendencias son; la tasa de crecimiento de la población, la distribución por edades, la movilidad; las variables educacionales, tales como nivel de formación general y específica, los sistemas de valores y creencias, y las normas de conducta que influyen en la demanda de las Superintendencias.

\section{Factores tecnológicos}

Respecto lo anterior, la tecnología es un factor determinante de la capacidad competitiva de las Superintendencias enfocada a la satisfacción del interés público, entendiéndose que se encuentran insertas en la nueva gestión pública, que radica en una serie de reformas administrativas que involucran una mejor gestión, una mayor orientación hacia el cliente, bajo las estrategias de informar y escuchar al cliente para comprender lo que quieren los ciudadanos, y responder con buenos servicios.

\section{ANÁLISIS DEL ENTORNO ESPECÍFICO}

\section{Competidores potenciales y productos sustitutos}

No existen competidores potenciales y productos sustitutos, debido a que su función es única en cada una de las áreas en que desarrollan sus acciones, es decir, en el ámbito de los servicios sanitarios, pensiones, salud, valores y seguros, casino de juegos, combustible y electricidad, servicio social y banco e instituciones financieras. 


\section{Proveedores}

En relación a los proveedores, su poder de negociación es bajo, debido principalmente a que las Superintendencias se encuentran insertas dentro del sector público y las adquisiciones de bienes y servicios de consumo como la adquisición de activos no monetarios, se rigen por la ley №19.886 de bases sobre contratos administrativos de suministro y prestación de servicios y por el decreto $\mathrm{N}^{\circ} 250$ que aprueba el reglamento de la ley antes señalada de fecha 24 de septiembre de 2009.

\section{Clientes}

El poder de negociación de los clientes es bajo, principalmente por la función de súper vigilancia y fiscalización que desarrollan las Superintendencias, en donde no existe un intercambio de dinero por los servicios proporcionados, la amenaza de que los clientes puedan integrarse hacia atrás es nula y no existe información privilegiada, respecto de la demanda, precios de mercado, costos de proveedor, entre otros, que les eleve su importancia.

\section{ANÁLISIS INTERNO}

En su mayoría las superintendencias realizan análisis interno consistente en identificar la identidad de la empresa, su perfil estratégico, cadena de valor, análisis FODA. Las herramientas más utilizadas para realizar los análisis internos y externos, consisten en la cadena de valor, perfil estratégico de la empresa, perfil estratégico del entorno, análisis de las fuerzas competitivas, análisis FODA, entrevistas a stakeholders y clientes internos. La tabla 4 indica las Superintendencias que llevan a cabo dicho análisis:

Tabla 4: Detalle del Análisis Interno y Externo de las Superintendencias.

\begin{tabular}{|c|c|c|c|c|c|c|c|}
\hline \multicolumn{7}{|c|}{ Realiza un análisis interno y externo para el desarrollo de la DE } \\
\hline SBIF & SCJ & SEC & SIP & SIS & SUSESO & SISS & SVS \\
\hline 0 & 0 & 0 & 0 & 0 & 0 & 1 & 1 \\
\hline
\end{tabular}

\section{FORMULACIÓN DE ESTRATEGIAS}

\section{Estrategia Corporativa}

Al respecto las Superintendencias del Estado de Chile, poseen estrategias de expansión, particularmente las de penetración de mercado y desarrollo de productos. La estrategia de penetración de mercado, se distingue porque las Superintendencias tratan de incrementar sus servicios que dicen relación con fiscalización, supervisión, respuestas a consultas y reclamos, atención de público, publicaciones, estudios técnicos y normativos, autorizaciones y registros, proposición de modificaciones legales y normativas y/o dictámenes de respuestas a las reclamaciones de usuarios, entre otros, dirigiéndose a sus usuarios actuales o bien intentando incrementar su número, dentro de la misma área geográfica, que corresponde a todo el territorio nacional de Chile. La estrategia de desarrollo de productos y/o servicios, se aprecia porque las Superintendencias se mantienen en el mercado actual, pero mejorando sus servicios, con características nuevas y diferentes que permitan optimizar la realización de su función, modificaciones que pueden ser accesorias, ligeras o sustanciales, desarrollo que se puede conseguir introduciendo innovaciones tecnológicas en los productos tradicionales, tanto de tipo incremental como radical.

\section{Estrategia Competitiva}

Como las Superintendencias de Chile son organismos autónomos con personalidad jurídica y patrimonio propio que representan al Estado en el ejercicio de las funciones de súper vigilancia y fiscalización, no poseen ventajas competitivas de coste o de diferenciación, sino que identifican productos estratégicos, que deben alcanzar determinados indicadores de gestión que van en función con los objetivos estratégicos de las instituciones. La pregunta del cuestionario relacionada con la definición de las estrategias, los resultados fueron los siguientes, ver tabla 5:

Tabla 5: Definición de las Estrategias de las Superintendencias Chilenas

\begin{tabular}{|c|c|c|c|c|c|c|c|}
\hline \multicolumn{7}{|c|}{ Definición de las Estrategias } \\
\hline SBIF & SCJ & SEC & SIP & SIS & SUSESO & SISS & SVS \\
\hline 0 & 0 & 0 & 1 & 0 & 0 & 0 & 0 \\
\hline
\end{tabular}


La mayoría de las Superintendencias informan que las estrategias están claramente definidas, y respecto al conocimiento de éstas por parte de toda la organización, sólo un $75 \%$ dijo estar de acuerdo.

\section{IMPLEMENTACIÓN DE LAS ESTRATEGIAS}

En relación a la factibilidad de las estrategias, todas las superintendencias han implementado una unidad de control de gestión o funcionarios a cargo de llevar a cabo la implementación y puesta en práctica de los procedimientos necesarios para alcanzar los indicadores de gestión, para cada uno de los productos estratégicos identificados, debido a que estos indicadores son los que permiten verificar el cumplimiento de los objetivos. Acerca de quiénes realizan el control, se observan en el tabla 6 .

Tabla 6: Realización del control estratégico de las Superintendencias de Chile.

\begin{tabular}{|l|c|c|c|c|c|c|c|c|}
\hline \multicolumn{8}{|c|}{ Realización del control estratégico } \\
\hline & SBIF & SCJ & SEC & SIP & SIS & SUSESO & SISS & SVS \\
\hline Directivos & 0 & 0 & 1 & 0 & 0 & 0 & 1 & 0 \\
\hline Auditores Internos & 1 & 1 & 1 & 1 & 1 & 1 & 1 & 1 \\
\hline Ambos & 1 & 1 & 0 & 1 & 1 & 1 & 1 & 1 \\
\hline Unidad de Gestión & 1 & 1 & 1 & 1 & 1 & 1 & 0 & 1 \\
\hline
\end{tabular}

La totalidad de las organizaciones consultadas afirmó que cuenta con un proceso de control de la Dirección Estratégica que es controlado en un $75 \%$ por sus directivos. Sobre las herramientas de control utilizadas, el $50 \%$ de los entrevistados señaló que usan el cuadro de mando integral (CMI), y las otras afirmaron que utilizan planillas de control e indicadores específicos. En las Tablas 7 y 8 se presentan dos ejemplos, de las formas en que utilizan los indicadores para medir los resultados de sus productos estratégicos, esto corresponde a las Superintendencias de Banco e Instituciones Financieras (SBIF) y a la Superintendencia de Casino de Juego (SCJ):

Tabla 7: Productos Estratégicos e Indicadores de la Superintendencia de Bancos e Instituciones Financieras.

\begin{tabular}{|l|l|}
\hline \multicolumn{1}{|c|}{ Producto Estratégico SBIF } & \multicolumn{1}{c|}{ Indicador } \\
\hline \multirow{3}{*}{ Información Consolidada } & Eficiencia/Producto: Monto de activos supervisados por funcionario \\
\cline { 2 - 3 } & $\begin{array}{l}\text { Eficacia/Producto: Variación anual de número de visitas al sitio Web de la } \\
\text { superintendencia }\end{array}$ \\
\hline $\begin{array}{l}\text { Atención directa a público y } \\
\text { procesamiento de reclamos }\end{array}$ & $\begin{array}{l}\text { Calidad/Producto: Variación anual de reclamos ingresados por escrito y que } \\
\text { son respondidos }\end{array}$ \\
\hline Publicaciones & $\begin{array}{l}\text { Eficacia/Producto: Variación anual de número de visitas al sitio Web de la } \\
\text { Superintendencia }\end{array}$ \\
\hline
\end{tabular}

Tabla 8: Productos Estratégicos e Indicadores de la Superintendencia de Casinos de Juegos.

\begin{tabular}{|c|c|}
\hline Producto Estratégico SCJ & Indicador \\
\hline \multirow{2}{*}{$\begin{array}{l}\text { Fiscalización de casinos de juego } \\
\text { en operación. }\end{array}$} & $\begin{array}{l}\text { Eficiencia/Producto: Promedio de fiscalizaciones por casino de juego, en el periodo } \\
\mathrm{t}\end{array}$ \\
\hline & $\begin{array}{l}\text { Calidad/Producto: Porcentaje de informes de operación mensual de casinos de } \\
\text { juego, revisados en un tiempo menor o igual a } 15 \text { días desde su fecha de } \\
\text { recepción en la Superintendencia, en el periodo t }\end{array}$ \\
\hline $\begin{array}{l}\text { Supervisión de la instalación de } \\
\text { nuevos casinos de juego. }\end{array}$ & $\begin{array}{l}\text { Eficacia/Producto: Promedio de visitas de supervisión por casino en } \\
\text { construcción, en el periodo t }\end{array}$ \\
\hline \multirow{2}{*}{$\begin{array}{l}\text { Respuesta a consultas y reclamos } \\
\text { de los visitantes a los casinos de } \\
\text { juego y público en general. }\end{array}$} & $\begin{array}{l}\text { Calidad/Producto } \\
\text { Porcentaje de consultas ingresadas por Oficina de Partes o a través del portal } \\
\text { web de la Superintendencia, respondidas en un tiempo menor o igual a } 30 \text { días, } \\
\text { en el periodo t }\end{array}$ \\
\hline & $\begin{array}{l}\text { Calidad/Producto } \\
\text { Porcentaje de reclamos ingresados por Oficina de Partes o a través del portal } \\
\text { web de la Superintendencia, respondidos en un tiempo menor o igual a } 90 \text { días, } \\
\text { en el periodo t }\end{array}$ \\
\hline $\begin{array}{l}\text { Evaluación de solicitudes } \\
\text { realizadas por las sociedades } \\
\text { operadoras, que requieren } \\
\text { presentación al Consejo } \\
\text { Resolutivo de la Superintendencia. }\end{array}$ & $\begin{array}{l}\text { Calidad/Producto } \\
\text { Porcentaje de informes con la evaluación de las solicitudes de las sociedades } \\
\text { operadoras, presentados al Consejo Resolutivo, en un tiempo menor o igual a } 30 \text { días } \\
\text { desde la fecha de ingreso de las solicitudes a la Superintendencia, en el periodo t }\end{array}$ \\
\hline
\end{tabular}




\section{CONCLUSIONES}

Las directrices estratégicas de las superintendencias en general, consideran los componentes necesarios que permiten una correcta formulación, a excepción de la dimensión de tiempo que debiera poseer la visión y las capacidades esenciales que requiere la misión. Se observa que las directrices estratégicas están desarrolladas principalmente por los directivos de las organizaciones en estudio.

En cuanto al análisis de la estructura organizacional de las Superintendencias, se observa que son de tipo matricial, por cuanto combinan dos estructuras de autoridad, que corresponden a las unidades por propósitos y los departamentos funcionales y multidivisionales, porque se basan en la agrupación de sus unidades por propósitos, como lo son sus distintas divisiones.

Por otra parte, en relación al entorno general de las Superintendencias, los factores más influyentes son político legales, toda vez que son instituciones públicas autónomas que se relacionan con el gobierno a través de los ministerios y el superintendente es nombrado por el presidente; socioculturales, debido a las variaciones en las tasas de crecimiento de la población que implica un aumento de los usuarios de los distintos productos estratégicos, y tecnológicos por el soporte tecnológico que han debido implementar para satisfacer el interés público y para otorgar los productos estratégicos de manera más eficiente y eficaz.

Sobre el análisis del entorno específico, se infiere que las Superintendencias no poseen competidores potenciales ni productos sustitutos por ser organismos autónomos con personalidad jurídica y patrimonio propio ejerciendo una función única de súper vigilancia y fiscalización en cada una de las áreas en que realizan su labor. Cabe indicar, que en general las Superintendencias realizan análisis interno y externo para el desarrollo de la Dirección Estratégica y para ello utilizan la cadena de valor, perfil estratégico de la empresa, perfil estratégico del entorno, análisis de las fuerzas competitivas, análisis FODA, entrevistas a stakeholders y clientes internos.

Respecto a las estrategias corporativas, corresponden a estrategias de expansión, específicamente a penetración de mercado y desarrollo de productos, por cuanto con su vocación de servicio público han buscado a través de sus páginas web una relación fluida con el público en general, aplicando por otra parte estándares de calidad y transparencia a sus actos, para garantizar el funcionamiento de la industria y la fe pública de las instituciones fiscalizadas. En relación a las estrategias competitivas, se concluye que debido a que las Superintendencias de Chile son organismos autónomos con personalidad jurídica y patrimonio propio que representan al Estado en el ejercicio de las funciones de supervisión y fiscalización, no poseen ventajas competitivas de coste o de diferenciación, sino que identifican productos estratégicos, a partir de sus objetivos, los que por medio de indicadores de desempeño traducidos en fórmulas de cálculo verifican si éstos son alcanzados.

En relación a la factibilidad de la estrategia, todas han implementado una unidad de control de gestión o funcionarios a cargo de llevar a cabo la implementación y puesta en práctica de los procedimientos necesarios para alcanzar los indicadores de gestión para cada uno de los productos estratégicos identificados. En cuanto a quiénes participan en el proceso de implementación de la Dirección Estratégica, en general es compartido entre los directivos y los trabajadores. Respecto al control estratégico, se observa que todas las Superintendencias cuentan con un proceso de control de la Dirección Estratégica, él que es ejercido por sus directivos principalmente y la herramienta de control que prevalece es el cuadro de mando integral.

\section{AGRADECIMIENTOS}

La autora agradece a la Dirección de Investigación de la Universidad del Bío-Bío, como también a las alumnas tesistas de la carrera de Ingeniería Comercial, Yeimmy González y Natalia Ceballos por su valiosa colaboración.

\section{REFERENCIAS}

Álvarez, A., Estrategia, Planificación y Control en la Empresa, Editorial Rama, 1aㅡ edición, Vol.1, pp. 1-328, Madrid, España (2007)

Bittel, L. y Ramsey, J., Enciclopedia del Management. Barcelona, Grupo Editorial Océano (1997)

Bueno, E., Dirección Estratégica de la Empresa, Metodología, Técnicas y Casos, Pirámide, 5ae edición, Vol.1, pp. 1-576, Madrid, España (1996) 
Cabanelas, J., Dirección de Empresas bases en un entorno abierto y dinámico, España, Ediciones Pirámide (1997)

Carnota, O., Curso de Administración para dirigentes, La Habana, Editorial Ciencias Sociales (1981)

David, F., Conceptos de Administración Estratégica, Pearson, 9ª edición, Vol.1, pp. 1-325, México (2003)

Eden, C. y Ackermann, F., Making strategy: The Journey of Strategic Mangement, Sage Publications, Londres (1998)

Freeman, R.E., Strategic Mangement: A Stakeholder Approach, Pitman, Boston, Massachusetts (1984)

García Falcón, J.M. y Alamo Vera, F.R., La Planificación Estratégica de las Universidades, Conserjería de Educación, Cultura y Deportes, Gobierno de Canarias (1998)

Hamel, G. y Prahalad, C.K., El Propósito Estratégico, Harvard-Deusto Business Review, № 41, pp. 75-94, Primer trimestre (1990)

Hitt, A., Ireland, D. y Hoskisson, E., Administración Estratégica. Conceptos, Competitividad y Globalización, Thompson, 6⿳a edición, Vol.1, pp. 1-396, México (1999)

Johnson, G., Scholes, K. y Whittington, R., Dirección Estratégica, Pearson Prentice Hall, $7^{a}$ edición, Vol.1, pp. 1-712, Madrid, España (2006)

Majluf, N. y Hax, A., Estrategias para el Liderazgo Competitivo, Granica, 1ª edición, Vol.1, pp. 1-538, Barcelona, España (1997)

Menguzzato, M. y Renau, P., La Dirección Estratégica de la Empresa, Ariel, Vol. 2, 1ㄹa edición, Vol.1, pp. 1441, Barcelona, España (1991)

Mintzberg, H. y Lampel, J., Reflecting on the Strategy Process, Sloan Management Review, Vol. 40, № 3, pp. 21-30, Spring (1999)

Navas, J. y Guerras, L., La Dirección Estratégica de la Empresa, Teorías y Aplicaciones, Civitas, 4aㅡ Edición, Vol.1, pp. 1-704, Madrid, España (2007)

Paredes, A.S., Manual planificación estratégica, Alide, 1르 edición, Vol.1, pp. 37, Lima, Perú (1996)

Porter, M.E., Competitive Strategy: Techniques for Analyzing Industries and Competitors, New York, Free Press (1980)

Porter, M., The Competitive Advantage of Nations, Free Press, New York (1990)

Rumelt, R.P., Shendel, D.E y Teece, D.J., Fundamental Issues in Strategy: A Research Agenda, Harvard Business School Press, 2ª edición, Vol.1, pp. 1-636, Boston, Estados Unidos (1994)

Schendel, D. y Hoferm C., Strategic Management, Little, Brown\& Company, 1aㅡ edición, Vol.1, pp. 1-538, Boston, Estados Unidos (1979)

Sepúlveda, M.A., La experiencia de modernización del Estado en Chile, XI Congreso Internacional del CLAD sobre la Reforma del Estado y de la Administración Pública, Ciudad de Guatemala, 7 - 10 Nov. (2006)

Thompson, S., Dirección y Administración Estratégicas, Mc Graw Hill, México (1999)

Wheelen, T., Hunger, D., Oliva, I., Administración Estratégica y Política de Negocios, 10aㅡ edición, Pearson Educación, México (2007) 